\title{
ASYMPTOTIC DEPENDENCE OF REINSURANCE AGGREGATE CLAIM AMOUNTS
}

\author{
ANA J. MATA* \\ Heriot-Watt University, United Kingdom
}

\begin{abstract}
In this paper we study the asymptotic behaviour of the joint distribution of reinsurance aggregate claim amounts for large values of the retention level under various dependence assumptions. We prove that, under certain dependence assumptions, for large values of the retention level the ratio between the joint distribution of the aggregate losses and the product of the marginal distributions converges to a constant value that only depends on the frequency parameters.
\end{abstract}

\section{KEYWORDS}

Dependent risks, excess of loss, reinsurance layers, multivariate Panjer recursion, asymptotic independence.

\section{INTRODUCTION}

Recently the importance of modelling dependent insurance and reinsurance risks has attracted the attention of actuarial practitioners and scientists. Even though classical theories have been developed under the assumption of independence between risks, there are practical cases where this assumption is not valid.

In a recent paper Embrechts, McNeil and Straumann (2001) wrote:

"Although insurance has traditionally been built on the assumption of independence and the law of large numbers has governed the determination of premiums, the increasing complexity of insurance and reinsurance products has led recently to increased actuarial interest in the modelling of dependent risks..."

Although the literature on dependence between risks in insurance portfolios is increasing rapidly, very few authors have applied these development to practical problems, for example reinsurance modelling.

In this paper we study the problem of dependence between risks from the reinsurer's point of view when he provides excess of loss cover for two dependent risks under different dependence assumptions. When the reinsurer undertakes excess of loss reinsurance for a portfolio, in particular for catastrophe excess of

* e-mail: ana.mata@cnare.com 
loss, the probability that a claim will impact the reinsurance layer is very small. Therefore, in many cases the correlation coefficient between aggregate claim amounts for the reinsurer becomes very small. We could then be tempted to think that the dependence between portfolios disappears as we look at the tail of the distribution and that, therefore, we could assume independence. It has been largely discussed in the literature that the linear correlation coefficient is not a satisfactory measure of dependence in the non-normal case, see, for example, Embrechts, McNeil and Straumann (2001).

In this paper we look at the effect of different dependence assumptions and their effect on the joint distribution of the aggregate claim amounts compared to the product of the marginal distributions when the retention or attachment is large (hence, the probability of a claim to the layer tends to zero). In Section 2, we describe a model used for insurance and reinsurance aggregate claim amounts that are subject to the same events. In Section 2.1, we discuss how to calculate the distribution of the sum of aggregate claim amounts under different dependence assumptions. In Section 3, we define a measure of asymptotic dependence. We use this concept to study the effect of large values of the retention level on the joint distribution of reinsurance aggregate losses under the dependence assumptions described in Section 2. Numerical illustrations and discussion of the results are presented in Sections 3.2 and 3.3.

\section{A DEPENDENCE MODEL FOR REINSURANCE AGGREGATE LOSSES}

In this section we describe a model that has been typically used in the actuarial literature to model insurance aggregate claim amounts that are exposed to the same events or claims. This model has been proposed, for example, in Sundt (1999) and Ambagaspitiya (1999) where they develop multivariate recursions to calculate the joint distribution of the aggregate claim amounts. We will assume that there are only two portfolios, however the results can be generalised for any number of risks or portfolios.

The Model: Two risks or portfolios are affected by the same events, therefore they are subject to the same frequency distribution. This model is the general model described in Sundt (1999). One of the most common applications of this model is, for example, catastrophe reinsurance where several portfolios are exposed to the same events. Also in fire insurance, the same fire can cause damage to neighbouring buildings or properties insured under different policies by the same insurer.

\section{Assumptions:}

1. Let $N$ be the total number of claims in a fixed period of time. It is assumed that $N$ belongs to the Panjer class of counting distribution, i.e. there exists constants $a$ and $b$ such that $N$ satisfies

$$
P(N=n)=\left(a+\frac{b}{n}\right) P(N=n-1) \quad \text { for } n=1,2, \ldots
$$


2. Let $\left\{X_{i}\right\}_{i \geq 1}$ and $\left\{Y_{i}\right\}_{i \geq 1}$ be sequences of i.i.d. random variables representing the claim amounts for each risk. We assume that $\left(X_{i}, Y_{i}\right)$ are i.i.d. pairs from a bivariate distribution.

3. $N$ is independent of $\left(X_{i}, Y_{i}\right) \forall i$.

Then the aggregate claim amounts for these risks are

$$
S_{1}=\sum_{i=1}^{N} X_{i} \quad \text { and } \quad S_{2}=\sum_{i=1}^{N} Y_{i}
$$

The claim amounts for each risk could have various interpretations:

1. From the primary insurer's point of view, the losses due to the $i$ th event $\left(X_{i}, Y_{i}\right)$ may be dependent or independent.

2. From the reinsurer's point of view $\left(X_{i}, Y_{i}\right)$ may represent excess of loss claims due to the same event from different underlying risks whose individual losses may be dependent or independent. In reinsurance, this model could also be used for reinsurance losses for two excess of loss layers from the same underlying risk. In this case the aggregate losses are dependent not only through the number of events, but also through the claim distribution for the primary risk.

\subsection{Joint distribution of dependent aggregate claim amounts}

Sundt (1999) and Ambagaspitiya (1999) developed multivariate recursions that allow us to calculate the joint distribution of the aggregate claim amounts under the assumptions of the model described in Section 2. As we discussed above, the individual claim amounts for each portfolio are not necessarily independent and we assume that they are integer-valued random variables. The joint probability function is given by $p(x, y)$ for $x=0,1,2, \ldots, y=0,1,2, \ldots$ in appropriate units.

The aggregate claim amounts are as given in formula (1), and the recursion for the joint distribution of $\left(S_{1}, S_{2}\right)$ is as follows:

$$
g\left(s_{1}, s_{2}\right)=\sum_{u=0}^{s_{1}}\left(a+\frac{b u}{s_{1}}\right) \sum_{v=0}^{s_{2}} p(u, v) g\left(s_{1}-u, s_{2}-v\right),
$$

for $s_{1}=1,2, \ldots, s_{2}=0,1,2, \ldots$

$$
g\left(s_{1}, s_{2}\right)=\sum_{v=0}^{s_{2}}\left(a+\frac{b v}{s_{2}}\right) \sum_{u=0}^{s_{1}} p(u, v) g\left(s_{1}-u, s_{2}-v\right),
$$

for $s_{1}=0,1,2, \ldots, s_{2}=1,2, \ldots$ See Sundt (1999).

In many cases the insurer/reinsurer would only be interested in calculating the distribution of the sum of the total losses for both risks. For example, if we are interested in calculating how much capital we must allocate (under some criteria) to each portfolio separately or to the combined portfolio, then we 
would be interested in the distribution of the sum of the corresponding aggregate claim amounts.

To calculate the distribution of the sum of dependent aggregate claim amounts under the assumptions of the model described above it is not necessary to calculate the joint distribution. In the next section we discuss in more detail how this is possible.

\subsection{Distribution of the sum of dependent aggregate claim amounts}

Under the assumptions of the dependence model described in Section 2 the sum of the aggregate claim amounts is given by:

$$
S=S_{1}+S_{1}=\sum_{i=1}^{N} X_{i}+\sum_{i=1}^{N} Y_{i}=\sum_{i=1}^{N}\left(X_{i}+Y_{i}\right) .
$$

Therefore if we can calculate the distribution of the sum $X_{i}+Y_{i}$ for each $i \geq 1$, then the distribution of $S$ can be calculated using Panjer recursion for univariate compound random variables. We denote $U_{i}=X_{i}+Y_{i}$.

Given the joint distribution of the individual claims for the $i$ th event, the distribution of $U_{i}$ is given by:

$$
P\left(U_{i}=u\right)=P\left(X_{i}+Y_{i}=u\right)=\sum_{m=0}^{u} P\left(X_{i}=u-m, Y_{i}=m\right), \quad \text { for } u=0,1, \ldots
$$

If $X_{i}$ and $Y_{i}$ are independent then $P\left(U_{i}=u\right)$ is given by the convolution of the marginal distributions.

When we consider two excess of loss layers from the same risk, e.g. $\left(m_{1}, m_{2}\right)$ and $\left(m_{2}, m_{3}\right)$, if $Z_{i}$ represents the claim amount due to the $i$ th event for the primary insurer, then the losses for the reinsurer are

$$
\begin{aligned}
& X_{i}=\min \left(\max \left(Z_{i}-m_{1}, 0\right), m_{2}-m_{1}\right) \quad \text { and } \\
& Y_{i}=\min \left(\max \left(Z_{i}-m_{2}, 0\right), m_{3}-m_{2}\right) .
\end{aligned}
$$

Hence, $X_{i}+Y_{i}$ represents the losses for the combined layer $\left(m_{1}, m_{3}\right)$, whose distribution can be easily calculated from the distribution of $Z_{i}$. However, Mata (2000) showed that for layers of the same risk that are subject to different aggregate conditions such as reinstatements and aggregate deductibles, the distribution of the sum of aggregate losses for two or more layers is not equivalent to the distribution of total aggregate losses for the combined layer. Therefore, the bivariate recursion given in formulae (2) and (3) must be used in these cases.

Example 1. Assume a reinsurer is considering to provide excess of loss cover for the following two layers: 10 xs 20 and $10 x s 30$ from any two risks (in appropriate units and currency). The reinsurer is given the following information about the underlying risks: 
1. Both primary risks are exposed to the same possible events of claims. $N$, the number of claims during the period of coverage, follows a Poisson distribution with parameter $\lambda=1$.

2. The individual claims for the $i$ th event for each primary risk, $X_{i}$ and $Y_{i}$, have the same marginal distribution. We assume that the claim size distribution follows a Pareto distribution with parameters $\alpha=3$ and $\beta=10$ and probability density function:

$$
f(x)=\frac{\alpha \beta^{\alpha}}{(\beta+x)^{\alpha+1}} \quad \text { for } x>0 .
$$

Therefore, for each event the reinsurer's claim amounts are:

$$
X_{i}^{R}=\min \left(\max \left(0, X_{i}-20\right), 10\right) \quad \text { and } \quad Y_{i}^{R}=\min \left(\max \left(0, Y_{i}-30\right), 10\right) \text {, }
$$

hence, the reinsurer's aggregate claim amounts are:

$$
S_{1}^{R}=\sum_{i=1}^{N} X_{i}^{R} \quad \text { and } \quad S_{2}^{R}=\sum_{i=1}^{N} Y_{i}^{R}
$$

Since we do not have any extra information about the individual claim amounts for each risk, there are many dependence structures that can be used in order to calculate the joint distribution of the individual claim amounts. Even if we were given the marginal distributions and the correlation coefficient there are several possibilities for the joint distribution of the individual claim amounts, see, for example, Embrechts, McNeil and Straumann (2001). Let us study the following three set ups:

(a) The individual claim amounts $X_{i}$ and $Y_{i}$ are independent.

(b) The individual claim amounts are dependent and their joint distribution follows a bivariate Pareto distribution with parameters $\left(\alpha, \beta_{1}, \beta_{2}\right)$ and joint probability density function

$$
f(x, y)=\frac{\alpha(\alpha+1)}{\beta_{1} \beta_{2}}\left(-1+\frac{x+\beta_{1}}{\beta_{1}}+\frac{y+\beta_{2}}{\beta_{2}}\right)^{-(\alpha+2)}
$$

In this example $\alpha=3, \beta_{1}=\beta_{2}=10$. For more details about the multivariate Pareto distribution see, for example, Mardia et al (1979).

(c) The layers belong to the same underlying risk in which case notice that they are consecutive layers.

Each of these set ups satisfies the assumptions of the model described in Section 2. It can be seen that the covariance between the aggregate claims amounts under the assumptions of the model presented in Section 2 is given by:

$$
\operatorname{Cov}\left(S_{1}, S_{2}\right)=E(N) \operatorname{Cov}\left(X_{i}, Y_{i}\right)+\operatorname{Var}(N) E\left[X_{i}\right] E\left[Y_{j}\right] \quad \text { for } i \neq j
$$




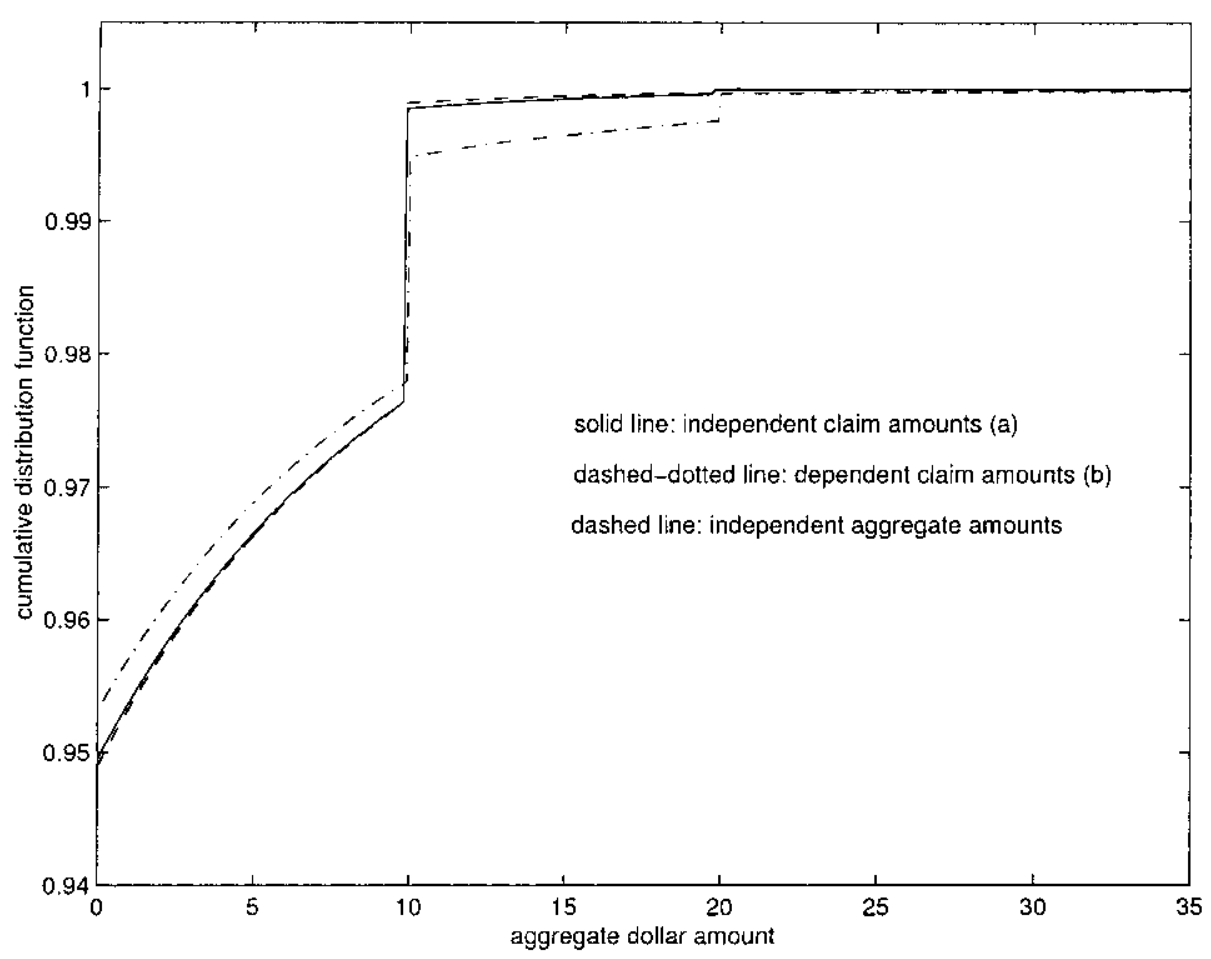

Figure 1: Distribution of $S_{1}^{R}+S_{2}^{R}$, cases (a) and (b).

It is interesting to note that the correlations calculated according to (5) are quite different from each other in the three cases considered in this example. The correlations are:

(a) $\rho\left(S_{1}^{R}, S_{2}^{R}\right)=0.019$

(b) $\rho\left(S_{1}^{R}, S_{2}^{R}\right)=0.206$

(c) $\rho\left(S_{1}^{R}, S_{2}^{R}\right)=0.761$

Figure 1 shows the c.d.f. of $S_{1}^{R}+S_{2}^{R}$ when the layers belong to different risks, i.e. dependence assumptions as in (a) and (b). The dashed line in Figure 1 represent the c.d.f. of $S_{1}^{R}+S_{2}^{R}$ when we assume these risks are completely independent, i.e. ignoring that both risks are exposed to the same claims. We observe that under the simplest dependence model (a), where the dependence arises only through the common number of events, the distribution of the total aggregate losses is very close to the distribution of total losses under the assumption of independence. However, when more complex dependence assumptions are built in, such as the bivariate Pareto claim distribution, the distribution of total aggregate losses is very different to the distribution under the independence assumption. In particular we notice that under the dependence assumption in (b) the tail of the distribution is significantly heavier than when independence is assumed. 


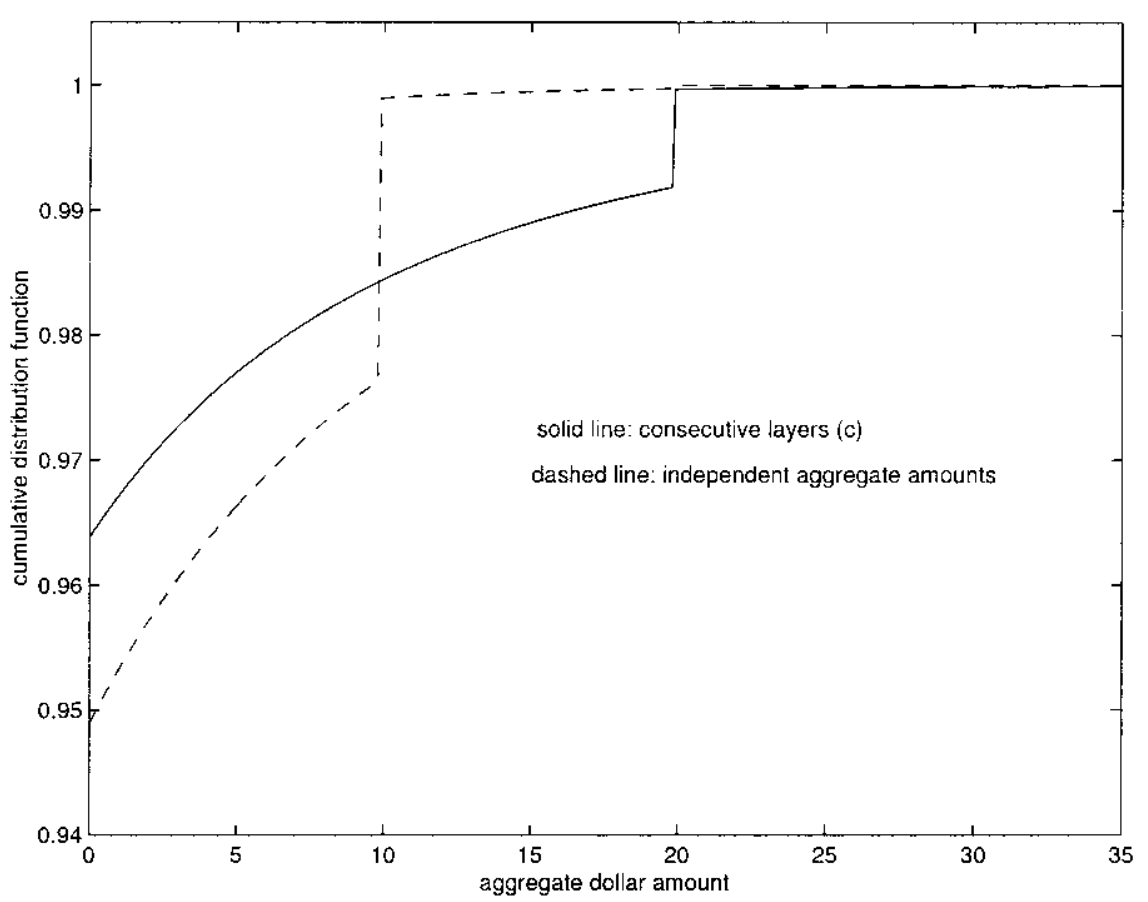

Figure 2: Distribution of $S_{1}^{R}+S_{2}^{R}$, cases (c).

Figure 2 shows the c.d.f. of $S_{1}^{R}+S_{2}^{R}$ when the layers belong to the same risk and the distribution of $S_{1}^{R}+S_{2}^{R}$ if independence is assumed. We notice that the distribution of total losses for consecutive layers would be totally mis-estimated if the dependence structure is ignored. This is of course due to the fact that when layers belong to the same risk there would be a positive claim in the second layer only when the claim for the first layers is a full loss. Hence, for layers of the same risk the claim amount dependence has more effect than the frequency dependence.

With this numerical example we have shown how different dependence assumptions may impact the distribution of total losses for the reinsurer. Therefore, by not taking into account how dependence arises one could mis-estimate the overall risk. This is of particular importance when pricing multi-layer excess of loss treaties, where dependence arises not only through he number of claims, but also through the claim size distribution.

\section{Asymptotic BeHAVIOUR OF DEPENDENT REINSURANCE AGGREGATE CLAIM AMOUNTS}

In the previous section we looked at the distribution of the sum of dependent aggregate claim amounts from the reinsurer's point of view. In Figures 1 and 2 
we observed the effect of different dependence structures that might be used to model reinsurance aggregate claim amounts.

Clearly, given the distribution of the individual claim amounts for the primary insurer and the distribution of the number of claims, the choice of retention level $M$ completely determines the distribution of the aggregate claim amounts for the reinsurer. For large values of the retention level, the probability of a claim to the reinsurance layer is small. Hence the probability of zero losses is very high, and therefore the correlation coefficient is small.

Based on the results showed in Figures 1 and 2, where we observed that under some dependence assumptions the distribution of total aggregate losses for the reinsurer is very close to the distribution of total aggregate losses if independence is assumed, it is our objective to give some insight to the following question:

Are the reinsurer's aggregate claim amounts from different but dependent risks approximately independent for large values of the retention levels?

In the next section we give some theoretical insight into the asymptotic behaviour of the distribution of the aggregate claim amounts for large values of the retention levels under different dependence assumptions.

\subsection{On measures of asymptotic independence for reinsurance aggregate claim amounts}

In order to provide some answers to the question outlined above we start by giving the definition of asymptotic independence which will be referred to in the remainder of the paper. For large values of the retention levels the probability of a non-zero loss for the reinsurer tends to zero. Hence we use the following definition of asymptotic independence.

Definition 1 Suppose two sequences of random variables $\left\{V_{n}\right\}$ and $\left\{W_{n}\right\}$ are dependent for each $n$. If these random variables satisfy

$$
\lim _{n \rightarrow \infty} \frac{P\left(V_{n} \in A, W_{n} \in B\right)}{P\left(V_{n} \in A\right) P\left(W_{n} \in B\right)}=1,
$$

for all sets $A$ and $B$ that have positive probability, then it is said that $V_{n}$ and $W_{n}$ are asymptotically independent. We will refer to the ratio in (6) as the dependence ratio.

We prove below that under certain dependence assumptions the reinsurance aggregate losses satisfy the condition given in (6) for some sets $A$ and $B$, but not for all sets. We set out below the assumptions we require to prove this result.

\section{Assumptions and notation:}

1. The primary insurance risks satisfy the dependence assumptions of the model described in Section 2, but we assume that the claim amounts for 
the $i$ th event are independent. Hence, the dependence structure arises only through the common number of events $N$.

2. Individual claim amounts for the primary insurer $X_{i}$ and $Y_{i}$ are integer-valued independent random variables that take values $x=0,1,2, \ldots$ and $y=0,1$, $2, \ldots$ in appropriate units. We assume that the probability functions of the individual claim amounts do not have fin§(ite upper limit. We denote $p_{1}(x)$ and $p_{2}(y)$ as the probability functions of the individual claim amounts for each portfolio.

3. The common number of claims, $N$, belongs to Panjer's class of counting distributions. Thus, $a$ and $b$ will represent the constants of Panjer's class.

4. We denote by $P_{N}(t)$ the probability generating function of $N$ which is defined as

$$
P_{N}(t)=E\left[t^{N}\right]
$$

5. Let $\left\{M_{1, n}\right\}_{n \geq 1}$ and $\left\{M_{2, n}\right\}_{n \geq 1}$ be sequences of integer numbers representing ting the retention levels of the excess of loss reinsurance for each risk. These sequences satisfy $M_{i, n}>M_{i, n-1}$ for $i=1,2$. For a given value of the retention, the reinsurer's claim amounts for the $i$ th event are:

$$
X_{i}^{R}\left(M_{1, n}\right)=\max \left(X_{i}-M_{1, n}, 0\right) \quad Y_{i}^{R}\left(M_{2, n}\right)=\max \left(Y_{i}-M_{2, n}, 0\right) .
$$

Therefore, the aggregate claim amounts for the reinsurer are:

$$
S_{1}^{R}\left(M_{1, n}\right)=\sum_{i=1}^{N} X_{i}^{R}\left(M_{1, n}\right) \quad \text { and } \quad S_{2}^{R}\left(M_{2, n}\right)=\sum_{i=1}^{N} Y_{i}^{R}\left(M_{2, n}\right) .
$$

The distribution functions of the aggregate losses are functions of the retention levels $\left\{M_{2, n}\right\}_{n \geq 1}$ and $\left\{M_{2, n}\right\}_{n \geq 1}$.

6. The retention levels are such that the reinsurer's aggregate claim amounts satisfy

$$
\lim _{n \rightarrow \infty} P\left(S_{i}^{R}\left(M_{i, n}\right)=0\right)=1, \quad \text { for } i=1,2 .
$$

7. The probability functions for the individual claim amounts for the reinsurer are $p_{1, n}(x)=P\left(X_{i}^{R}\left(M_{1, n}\right)=x\right)$ and $p_{2, n}(y)=P\left(Y_{i}^{R}\left(M_{2, n}\right)=y\right)$ for $x, y=$ $0,1,2, \ldots$,

8. We assume that the probability functions for the individual claim amounts satisfy

$$
\lim _{n \rightarrow \infty} \frac{p_{i}\left(x+M_{i, n}\right)}{p_{i}\left(y+M_{i, n}\right)}=C(x, y) \quad \text { for } x=1,2, \ldots, y
$$

for $y=1,2, \ldots$ where $C(x, y)$ is a constant that only depends on $x$ and $y$ and $0 \leq C(x, y)<\infty$. 
9. The probability functions for the aggregate claim amounts are $g_{1, n}\left(s_{1}\right)=$ $P\left(S_{1}^{R}\left(M_{1, n}\right)=s_{1}\right)$ and $g_{2, n}\left(s_{2}\right)=P\left(S_{2}^{R}\left(M_{2, n}\right)=s_{2}\right)$, for $s_{1}, s_{2}=0,1,2, \ldots$ We assume that $g_{i, n}\left(s_{i}\right)>0$ for $i=1,2$ and for $s_{1}, s_{2}=0,1,2, \ldots$

10. The joint probability function for the aggregate claim amounts is defined as $g_{n}\left(s_{1}, s_{2}\right)=P\left(S_{1}^{R}\left(M_{1, n}\right)=s_{1}, S_{2}^{R}\left(M_{2, n}\right)=s_{2}\right)$, for $s_{1}, s_{2}=0,1,2, \ldots$

Proposition 1. Under the assumptions outlined above the aggregate claim amounts for the reinsurer defined in (7) satisfy:

a) $\lim _{n \rightarrow \infty} \frac{g_{n}(0,0)}{g_{1, n}(0) g_{2, n}(0)}=1$

b) $\lim _{n \rightarrow \infty} \frac{P\left(S_{1}^{R}\left(M_{1, n}\right) \leq s_{1}, S_{2}^{R}\left(M_{2, n}\right) \leq s_{2}\right)}{P\left(S_{1}^{R}\left(M_{1, n}\right) \leq s_{1}\right) P\left(S_{2}^{R}\left(M_{2, n}\right) \leq s_{2}\right)}=1$ for all $s_{1}, s_{2}=0,1,2, \ldots$

c) $\lim _{n \rightarrow \infty} \frac{g_{n}\left(s_{1}, 0\right)}{g_{1, n}\left(s_{1}\right) g_{2, n}(0)}=1 \quad$ for $s_{1}=1,2, \ldots$

d) $\lim _{n \rightarrow \infty} \frac{g_{n}\left(0, s_{2}\right)}{g_{1, n}(0) g_{2, n}\left(s_{2}\right)}=1 \quad$ for $s_{2}=1,2, \ldots$

e) $\lim _{n \rightarrow \infty} \frac{g_{n}\left(s_{1}, s_{2}\right)}{g_{1, n}\left(s_{1}\right) g_{2, n}\left(s_{2}\right)}=1+\frac{1}{a+b} \quad$ for $s_{1}, s_{2}=1,2, \ldots$

Proof. The proof of this proposition is essentially an induction based proof. In order to avoid confusion with the details of the algebraic proof we leave the analytical proof for the Appendix and we concentrate in the interpretation of the results and the assumptions.

From the results shown in Proposition 1 we make the following remarks:

1. Note that for the Binomial, Poisson and Negative Binomial distribution it always holds that $a+b>0$. Hence, $1+\frac{1}{a+b}>1$. In other words, the dependence ratio is always greater than or equal to 1 .

2. The statement in $b$ ) implies that when we consider cumulative distributions we are including the value of zero which has a high probability for large values of the retention level. This result explains the behaviour observed in Figures 1 and 2 where we considered the cumulative distribution function of the sum of the reinsurer's aggregate claim amounts. In other words, if zero is included in the probability being evaluated the probability would tend to 1 due to assumption 6 above.

3. In Proposition 1 we assumed one of the simplest cases of dependence in insurance/reinsurance risks. Hence, under more complicated assumptions of dependence between risks the dependence ratio might converge to a different value. In the next section we compare numerically the asymptotic behaviour of the dependence ratio under various dependence assumptions. 
4. Statement e) shows cases where the ratio between the joint distribution and the product of the marginal distribution does not tend to 1 , which proves the fact that even under the simplest dependence assumption independence cannot be assumed. However, if $a+b$ takes large values, the limit would be close to 1 . For example, when $N$ follows a Poisson distribution with parameter $\lambda, a+b=\lambda$ which is the expected number of common events per unit of time. If we increase $\lambda$ we are increasing the dependence parameter as the expected number of common events becomes larger. Nevertheless, by increasing $\lambda$ the limit in e) would be closer to 1 which implies that the joint distribution is closer to the independent case. This shows how counter-intuitive results can be when the independence assumption is relaxed.

The following proposition shows another case when the result in e) also holds.

Proposition 2 Assume that two risks follow assumptions 1 and 3 of Proposition 1. Let $\left\{M_{1, n}\right\}$ and $\left\{M_{2, n}\right\}$ be sequences of real numbers representing the reinsurance retention levels for each portfolio. These sequences are such that the reinsurer's aggregate claim amounts satisfy the condition in (8) and that for each $n$

$$
P\left(S_{1}^{R}\left(M_{1, n}\right)=0\right)=P\left(S_{2}^{R}\left(M_{2, n}\right)=0\right)=\alpha_{n}
$$

Then, the reinsurance aggregate claim amounts defined in (7) satisfy

$$
\lim _{n \rightarrow \infty} \frac{P\left(S_{1}^{R}\left(M_{1, n}\right)>0, S_{2}^{R}\left(M_{2, n}\right)>0\right)}{P\left(S_{1}^{R} M_{1, n}>0\right) P\left(S_{2}^{R}\left(M_{2, n}\right)>0\right)}=1+\frac{1}{a+b} .
$$

Proof. We give detailed analytical proof of this result in the Appendix.

Note that the assumptions for Proposition 2 are more general than the assumptions we made for Proposition 1. Since the result in Proposition 2 refers to the joint survival function evaluated at zero, the claim size distribution could have a continuous density function. Also the retention levels are not required to be sequences of integer values, it is enough that $\left\{M_{1, n}\right\}_{n \geq 1}$ and $\left\{M_{2, n}\right\}_{n \geq 1}$ are sequences of real numbers such that:

$$
\lim _{n \rightarrow \infty} P\left(S_{i}^{R}\left(M_{i, n}\right)=0\right)=1 \quad \text { for } i=1,2
$$

For Proposition 2, we assumed that both aggregate claim amounts for the reinsurer have the same probability of being zero. Nevertheless, in Example 2 we show that this is not a necessary assumption. It seems to be sufficient that when $n$ tends to infinity the probabilities of being zero tend to one. We discuss this in more detail in Example 2. 


\subsection{Numerical illustrations}

It is our objective in this section to illustrate numerically the results shown in previous section. We compare numerically the behaviour of the dependence ratio as defined in (6) under all dependence assumptions described in Example 1 above.

Example 2. Assume that two insurance portfolios follow the distributional assumptions as in Example 1. For each risk the reinsurer takes a layer of size 10 with deductibles $M_{1, n}=n$ and $M_{2, n}=10+n$ for $n=0,1,2, \ldots$. Therefore, for each event the reinsurer receives claims for the following amounts

$$
\begin{aligned}
& X_{i}^{R}\left(M_{1, n}\right)=\min \left(\max \left(0, X_{i}-n\right), 10\right) \text { and } \\
& Y_{i}^{R}\left(M_{2, n}\right)=\min \left(\max \left(0, Y_{i}-10-n\right), 10\right) .
\end{aligned}
$$

We consider the three set ups as described in Example 1. In this example $N$ follows a Poisson distribution with parameter $\lambda=1$. Hence, it follows that $1+\frac{1}{a+b}=2$.

Figure 3 shows the dependence ratio $\frac{P\left(S_{1}^{R}\left(M_{1, n}\right)>0, S_{2}^{R}\left(M_{2, n}\right)>0\right)}{P\left(S_{1}^{R}\left(M_{1, n}\right)>0\right) P\left(S_{2}^{R}\left(M_{2, n}\right)>0\right)}$ as $n \rightarrow \infty$. We observe that the asymptotic behaviour of the joint survival function at zero is very different under the three dependence assumptions. In the case of independent claim amounts, the dependence ratio converge to $1+\frac{1}{a+b}$ as shown in Proposition 2. However, when the claim amounts are dependent, as in cases (b) and (c), the dependence ratio tends to infinity. We also notice that for layers of the same risk the dependence ratio goes to infinity faster since

$$
P\left(S_{1}^{R}\left(M_{1, n}\right)>0, S_{2}^{R}\left(M_{2, n}\right)>0\right)=P\left(S_{2}^{R}\left(M_{2, n}\right)>0\right)
$$

and therefore,

$$
\lim _{n \rightarrow \infty} \frac{P\left(S_{1}^{R}\left(M_{1, n}\right)>0, S_{2}^{R}\left(M_{2, n}\right)>0\right)}{P\left(S_{1}^{R}\left(M_{1, n}\right)>0\right) P\left(S_{2}^{R}\left(M_{2, n}\right)>0\right)}=\lim _{n \rightarrow \infty} \frac{1}{\left(S_{1}^{R}\left(M_{1, n}\right)>0\right)}=\infty .
$$

Figure 4 shows the behaviour of $\frac{g_{n}(0,1)}{g_{1, n}(0) g_{2, n}(1)}$ for large values of the retention $n$. We note that for layers of the same risk the dependence ratio has a constant value of zero since it is not possible that the second layer takes a positive value if the first layer is zero. However, for layers of separate risks the dependence ratio tends to one. We also observe that in the case of layers from different underlying risks with dependent claim amounts the dependence ratio converges to one, but slower than in the case of independent claims amounts. 


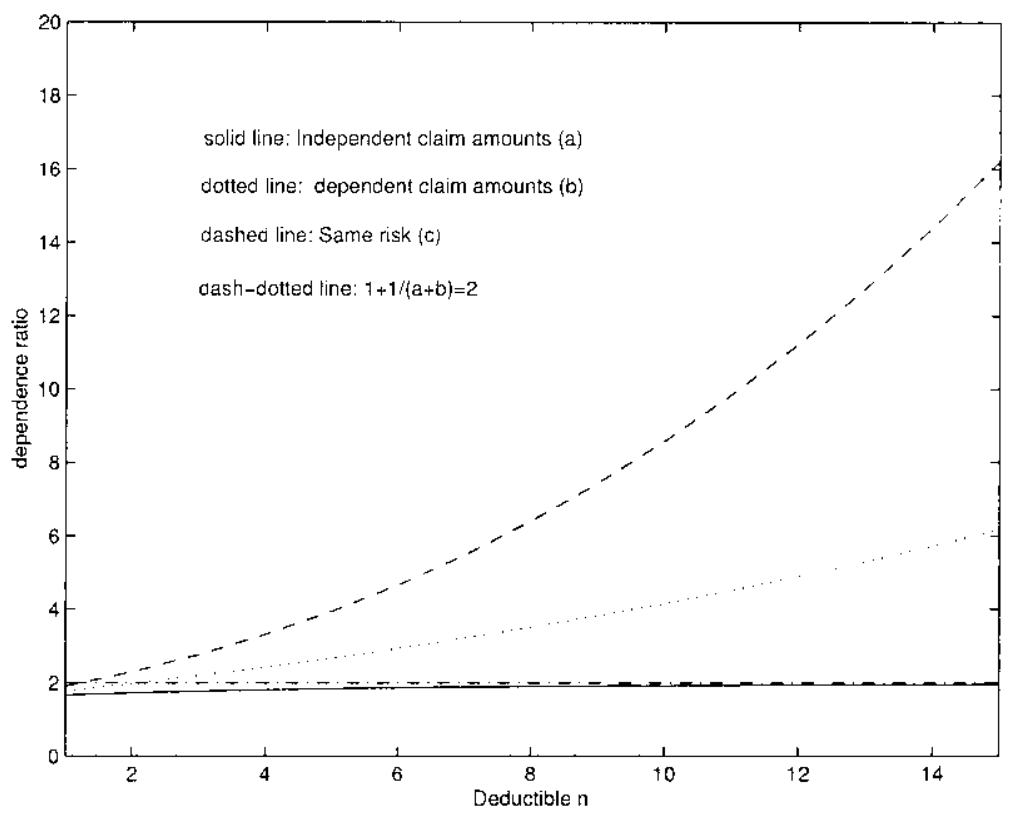

FIGURE 3: Behaviour of $\frac{P\left(S_{1}^{R}\left(M_{1, n}\right)>0, S_{2}^{R}\left(M_{2, n}\right)>0\right)}{P\left(S_{1}^{R}\left(M_{1, n}\right)>0\right) P\left(S_{2}^{R}\left(M_{2, n}\right)>0\right)}$, for Poisson $(\lambda=1)$

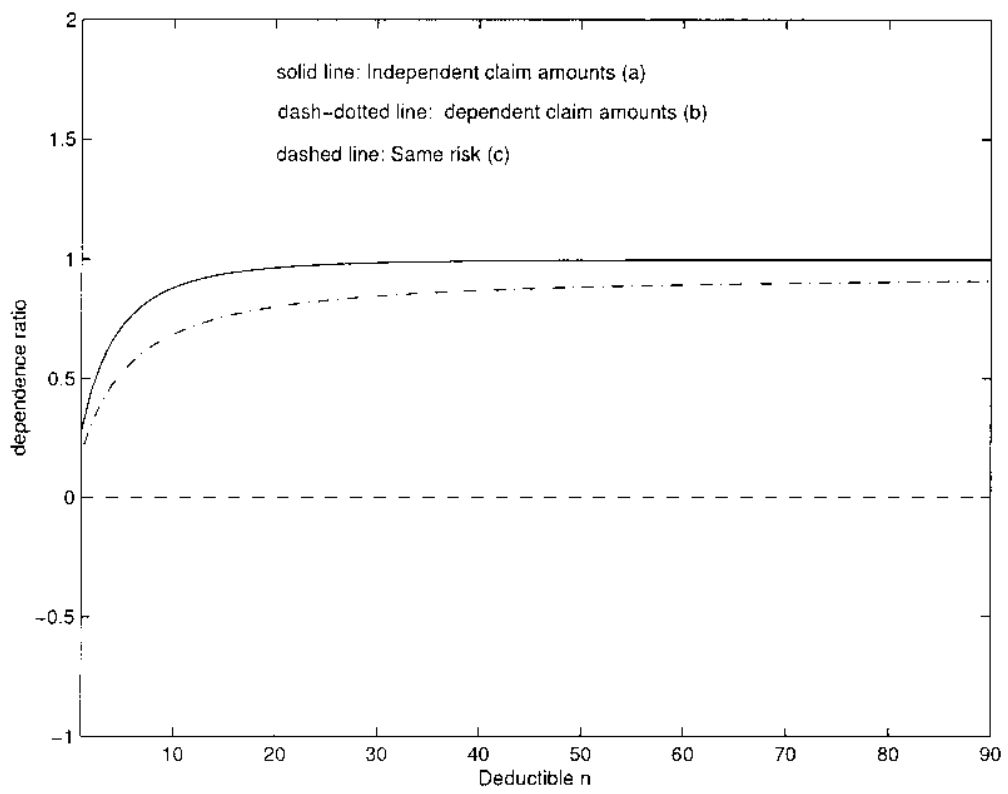

FIGURE 4: Behaviour of $\frac{g_{n}(0,1)}{g_{1, n}(0) g_{2, n}(1)}$, for Poisson $(\lambda=1)$ 


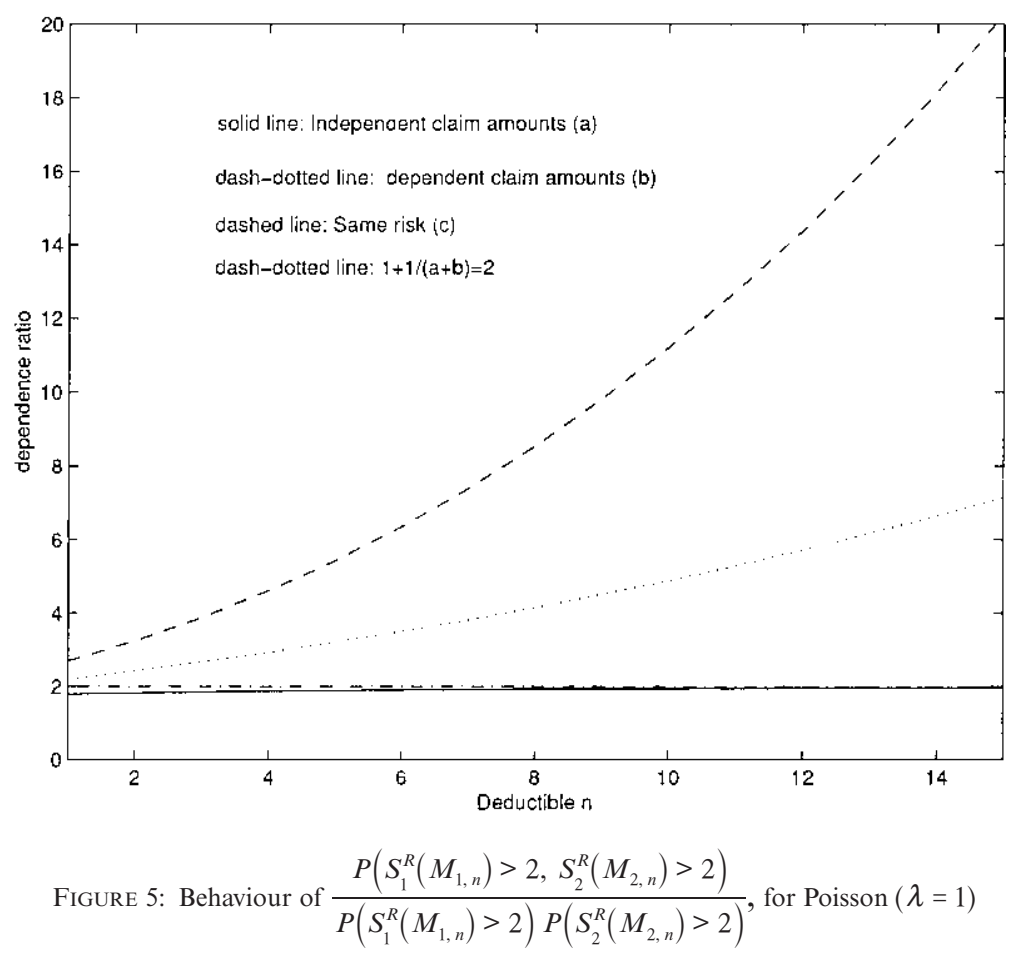

Figure 5 shows the behaviour of

$$
\frac{P\left(S_{1}^{R}\left(M_{1, n}\right)>2, S_{2}^{R}\left(M_{2, n}\right)>2\right)}{P\left(S_{1}^{R}\left(M_{1, n}\right)>2\right) P\left(S_{2}^{R}\left(M_{2, n}\right)>2\right)} \quad \text { as } n \rightarrow \infty
$$

We notice that the asymptotic behaviour of joint survival functions for $s_{1}, s_{2}>0$ (in this case $s_{1}=s_{2}=2$ ) is very similar to the asymptotic behaviour of the joint survival functions for $s_{1}=s_{2}=0$. In the case of independent claim amounts the dependence ratio for the joint survival function also converges to $1+\frac{1}{a+b}$ as shown in Proposition 2 for $s_{1}=s_{2}=0$.

\subsection{Comments on the assumptions in Section 3.1 in practical applications}

The results shown in Section 3.1 are a step towards a better understanding of the effect of dependence between reinsurance risks that have small probabilities of large losses. In order to get a better understanding of the results of Propositions 1 and 2 one has to look closely to each of the assumptions made. We discuss below the relevance of each assumption. 
1. Assumption 5: Note that in Example 2 we have assumed that the excess of loss layers for the reinsurer have a finite upper limit equal to 10 . Therefore, assumption 5 does not seem to be a restriction in practical terms. However, the assumption that the reinsurer takes excess of loss layers with infinite limit facilitated the analytical proof.

2. Assumption 6: It is not unreasonable to assume that for non proportional reinsurance the retention or deductible is such that the probability of claims affecting the reinsurer is very small. This is a typical assumption in practice.

3. Assumption 8: We assumed that the probability function is such that $\lim _{M \rightarrow \infty} \frac{f(x+M)}{f(y+M)}=C(x, y)$ for $0<x \leq y$, where $0 \leq C(x, y)<\infty$ and analytically this is the key assumption for the proof of Proposition 1. Although this property seems to be related to the theory of slowly or regularly varying functions (see, for example, Embrechts, Mikosh and Klüppelberg (1997)) it is in fact a weaker condition as some density functions satisfy assumption 8 but are not regularly varying functions. For example, the Exponential distribution satisfies the condition in assumption 8, however its probability density function is not a regularly varying function. On the other hand, the density function of a Pareto distribution is a regularly-varying function and it also satisfies the condition in assumption 8 . The condition in assumption 8 is satisfied by most of the continuous loss distributions used to model insurance/reinsurance losses, such as: Exponential, Gamma, Log-normal, Pareto and Generalised Pareto. The Normal distribution does not satisfy this property as it can be seen that $\lim _{m \rightarrow \infty} \frac{f(x+m)}{f(m+y)}=\infty$. In practical cases the loss distributions for insurance claims are usually skewed and heavy-tailed, and therefore, the Normal distribution is not a reasonable loss distribution for practical use.

4. Proposition 2. In this proposition we assumed that both aggregate claim amounts have the same probability of being zero. Example 2 shows that this seems not to be a restriction. In fact in Example 2 the layers are such that for each $n$ we have

$$
P\left(S_{1}^{R}\left(M_{1, n}\right)=0\right)<P\left(S_{2}^{R}\left(M_{2, n}\right)=0\right) .
$$

However, for any $\epsilon>0$ there is $M$ such that for $n \geq M$

$$
P\left(S_{2}^{R}\left(M_{2, n}\right)=0\right)-P\left(S_{1}^{R}\left(M_{1, n}\right)=0\right)<\epsilon,
$$

and as $n$ tends to infinite both probabilities tend to one.

\section{Conclusions}

Modelling dependencies between risks has become an area of increased research interest in actuarial science. Although many authors have emphasized 
the importance of differentiating between correlation and dependence, in practice, when one thinks of dependencies, inevitably the correlation coefficient is the first thing that comes to mind.

The numerical examples in this paper showed that even when the correlation coefficient becomes small dependence cannot be ignored. Failure to identify dependence between risks may lead to underestimation of the overall risk. This is particularly relevant when pricing risks or managing aggregation of risk exposure.

Throughout this paper we have looked at dependencies from the reinsurer's point of view where there is a very small probability of very large losses. Loosely speaking, the main result states that for large values of the retention levels the dependence ratio converges to a constant defined by the frequency distribution. This constant is always greater than or equal to one. Intuitively, if the aggregate losses are dependent only through the number of events, one would be inclined to think that if the expected number of events increases then the dependence becomes stronger. However, we showed that when the number of events follows a Poisson distribution the larger the expected number of events the joint distribution of aggregate losses gets closer to the product of the marginal distributions which is the distribution of independent aggregate claim amounts.

Modelling dependencies is an area with a vast possibility for research. It is of particular importance to extend the ideas presented in this paper of comparing the joint distribution with the product of the marginal distributions for more general right tail dependence models, for example by looking at multivariate extreme value distributions or extreme value copulas. This comparison is always helpful in practice when often the practical actuary is interested in the impact of making simplified assumptions to facilitate the implementation of new models and techniques.

\section{ACKNOWLEDGEMENTS}

This research was carried out while the author was a research student in the Department of Actuarial Mathematics and Statistics at Heriot-Watt University, Edinburgh. The author would like to thank Professor Howard Waters for his invaluable advise, helpful comments and patience throughout the numerous versions of this paper. Many thanks also to the helpful comments of the anonymous referees and my colleague Mr. Parr Schoolman that significantly helped to improve the presentation of the results. The author is also thankful for the financial support of The British Council and FUNDAYACUCHO from Venezuela.

\section{REFERENCES}

AmbagaspitiYa, R.S. (1999) On the distribution of two classes of correlated aggregate claims. Insurance: Mathematics and Economics 24:301-308 
Embrechts, P., McNeil, A.J. and Straumann, D. (2001) Correlations and dependency in risk management: properties and pitfalls In Risk Management: Value at Risk and Beyond, ed. by M. Dempters and H.K. Moffat. Cambridge University Press.

Embrechts, P., Mikosh, T. and KlüPpelberg, C. (1997) Modelling Extremal Events for Insurance and Finance. Springer, Berlin.

Mardia, K.V., Kent, J.T. and Bibby, J.M. (1979) Multivariate Analysis. Academic Press, London.

MatA, A.J. (2000) Pricing excess of loss reinsurance with reinstatemets. ASTIN Bulletin 30(2): 349-368.

PANJER, H.H. (1981) Recursive evaluation of a family of compound distributions. ASTIN Bulletin 12(1): 21-26.

SundT, B. (1999) On multivariate Panjer recursions. ASTIN Bulletin 29(1): 29-46. 


\section{ApPendix: AnAlytical proof of Propositions 1 And 2}

This Appendix gives the analytical proof of Propositions 1 and 2 in Section 3.1.

Proposition 1: Under the assumptions outlined in Section 3.1. the aggregate claim amounts for the reinsurer defined in (7) satisfy:

a) $\lim _{n \rightarrow \infty} \frac{g_{n}(0,0)}{g_{1, n}(0) g_{2, n}(0)}=1$

b) $\lim _{n \rightarrow \infty} \frac{P\left(S_{1}^{R}\left(M_{1, n}\right) \leq s_{1}, S_{2}^{R}\left(M_{2, n}\right) \leq s_{2}\right)}{P\left(S_{1}^{R}\left(M_{1, n}\right) \leq s_{1}\right) P\left(S_{2}^{R}\left(M_{2, n}\right) \leq s_{2}\right)}=1 \quad$ for all $s_{1}, s_{2}=0,1,2, \ldots$

c) $\lim _{n \rightarrow \infty} \frac{g_{n}\left(s_{1}, 0\right)}{g_{1, n}\left(s_{1}\right) g_{2, n}(0)}=1 \quad$ for $s_{1}=1,2, \ldots$

d) $\lim _{n \rightarrow \infty} \frac{g_{n}\left(0, s_{2}\right)}{g_{1, n}(0) g_{2, n}\left(s_{2}\right)}=1 \quad$ for $s_{2}=1,2, \ldots$

e) $\lim _{n \rightarrow \infty} \frac{g_{n}\left(s_{1}, s_{2}\right)}{g_{1, n}\left(s_{1}\right) g_{2, n}\left(s_{2}\right)}=1+\frac{1}{a+b} \quad$ for $s_{1}, s_{2}=1,2, \ldots$

\section{Proof.}

Reminder: in what follows $n$ represents the indexation of the retention level that are increasing sequences (tending to infinity) as defined in the assumptions in Section 3.1.

a) Since the aggregate claim amounts satisfy the condition (8) in assumption 6 , we have that $\lim _{n \rightarrow \infty} g_{i, n}(0)=1$ for $i=1,2$. This also implies that $\lim _{n \rightarrow \infty} p_{i, n}(0)=1$ for $i=1,2$. The joint probability of being zero is given by $g_{n}(0,0)=$ $P_{N}\left(p_{1, n}(0) p_{2, n}(0)\right)$, where $P_{N}(t)$ is the probability generating function of $N$. Therefore from assumption 6 it also holds that $\lim _{n \rightarrow \infty} g_{n}(0,0)=1$. Then we directly obtain the result in a).

b) From a) we have that the joint probability of being zero tends to one as well as the probability of each aggregate claim amount being zero. Hence, the result in b) follows directly since we are considering cumulative probabilities which include the value of zero.

c) Since we have assumed that the random variables are integer-valued we can evaluate the joint distribution of the aggregate claim amounts using the bivariate recursion proposed by Sundt (1999) defined in formulae (2) and (3). We will prove the statement in c) by induction and we do the basic step for $s_{1}=1$. 


$$
\begin{aligned}
\lim _{n \rightarrow \infty} \frac{g_{n}(1,0)}{g_{1, n}(1) g_{2, n}(0)} & =\lim _{n \rightarrow \infty} \frac{\frac{1}{1-a p_{1, n}(0) p_{2, n}(0)}(a+b) p_{1, n}(1) p_{2, n}(0) g_{n}(0,0)}{\left(\frac{1}{1-a p_{1, n}(0)}(a+b) p_{1, n}(1) g_{1, n}(0)\right) g_{2, n}(0)} \\
& =\frac{1-a}{1-a},
\end{aligned}
$$

where the last inequality is due to the result in a). The limit above is equal to 1 , only when $a \neq 1$. For the Poisson, Negative Binomial and Binomial distributions $a$ takes the values: $a=0, a=(1-p)$ and $a=-p /(1-p)$, respectively. Hence $a \neq 1$ for the three distributions that belong to Panjer's class. Therefore the limit above is equal to 1 . We now state the inductive hypothesis: Let us assume that the result in c) holds for all the pairs $\left(s_{1}, 0\right)$ such that $s_{1}=1,2, \ldots, X$, then we have to prove that it is also true for $(X+1,0)$ (note that $X$ in this context does not represent a random variable, it is an index in the induction proof). From the recursion in (2) we have

$g_{n}(X+1,0)=\frac{p_{2, n}(0)}{1-a p_{1, n}(0) p_{2, n}(0)} \sum_{u=1}^{X+1}\left(a+\frac{b u}{X+1}\right) p_{1, n}(u) g_{n}(X+1-u, 0)$

Using Panjer's univariate recursion for $g_{1, n}(X+1)$, we have

$$
\frac{g_{n}(X+1,0)}{g_{1, n}(X+1) g_{2, n}(0)}=
$$

$$
\frac{\frac{p_{2, n}(0) g_{2, n}(0)\left[1-a p_{1, n}(0)\right]}{1-a p_{1, n}(0) p_{2, n}(0)} \sum_{u=1}^{X+1}\left(a+\frac{b u}{X+1}\right) p_{1, n}(u) g_{1, n}(X+1-u) \frac{g_{n}(X+1-u, 0)}{g_{1, n}(X+1-u) g_{2, n}(0)}}{g_{2, n}(0) \sum_{u=1}^{X+1}\left(a+\frac{b u}{X+1}\right) p_{1, n}(u) g_{1, n}(X+1-u)}
$$

Since $\lim _{n \rightarrow \infty} p_{1, n}(0)=\lim _{n \rightarrow \infty} p_{2, n}(0)=1$, and using the inductive hypothesis, for any $\epsilon>0$ there exists $K$ such that for $n \geq K$

$$
1-\epsilon<\frac{p_{2, n}(0)\left[1-a p_{1, n}(0)\right]}{1-a p_{1, n}(0) p_{2, n}(0)} \frac{g_{n}(X+1-u, 0)}{g_{1, n}(X+1-u) g_{2, n}(0)}<1+\epsilon,
$$

for $u=1,2, \ldots, X+1$. Hence for $n \geq K$

$$
1-\epsilon<\frac{g_{n}(X+1,0)}{g_{1, n}(X+1) g_{2, n}(0)}<1+\epsilon,
$$

which proves c).

d) The prove of the statement in d) follows the same argument as c) but using the recursion in formula (3) instead of (2).

e) To prove the statement in e) we will use the results in a), c) and d). We start by proving the result for $s_{1}=1$ and $s_{2}=1$. Using the recursion in formula (2) we have 


$$
\begin{aligned}
& \lim _{n \rightarrow \infty} \frac{g_{n}(1,1)}{g_{1, n}(1) g_{2, n}(1)}= \\
& \lim _{n \rightarrow \infty} \frac{1}{1-a p_{1, n}(0) p_{2, n}(0)} \\
& {\left[\frac{a p_{1, n}(0) p_{2, n}(1) g_{n}(1,0)}{g_{1, n}(1) g_{2, n}(1)}+\frac{(a+b) p_{1, n}(1) \sum_{v=0}^{1} p_{2, n}(v) g_{n}(0,1-v)}{g_{1, n}(1) g_{2, n}(1)}\right]}
\end{aligned}
$$

In the limit above we have three terms, we will analyse each term separately. Using Panjer's univariate recursion for $g_{2, n}(1)$ the limit for the first term can be calculated as follows:

$$
\lim _{n \rightarrow \infty} \frac{a p_{1, n}(0) p_{2, n}(1) g_{2, n}(0)}{\frac{1}{1-a p_{2, n}(0)}(a+b) p_{2, n}(1) g_{2, n}(0)} \frac{g_{n}(1,0)}{g_{1, n}(1) g_{2, n}(0)}=\frac{a(1-a)}{a+b},
$$

since from part c) we know that $\lim _{n \rightarrow \infty} \frac{g_{n}(1,0)}{g_{1, n}(1) g_{2, n}(0)}=1$. For the second term we use the result in d) and Panjer's univariate recursion for $g_{1, n}(1)$, therefore the limit for the second term is

$$
\lim _{n \rightarrow \infty} \frac{(a+b) p_{1, n}(1) p_{2, n}(0) g_{1, n}(0)}{\frac{1}{1-a p_{1, n}(0)}(a+b) p_{1, n}(1) g_{1, n}(0)} \frac{g_{n}(0,1)}{g_{1, n}(0) g_{2, n}(1)}=1-a .
$$

And finally for the third term we use the result in a) together with Panjer's univariate algorithms for $g_{1, n}(1)$ and $g_{2, n}(1)$, hence the limit is

$$
\lim _{n \rightarrow \infty} \frac{(a+b) p_{1, n}(1) g_{1, n}(0) p_{2, n}(1) g_{2, n}(0)}{g_{1, n}(1) g_{2, n}(1)} \frac{g_{n}(0,0)}{g_{1, n}(0) g_{2, n}(0)}=(1-a)\left(\frac{1-a}{a+b}\right)
$$

Putting these three results together we obtain

$$
\lim _{n \rightarrow \infty} \frac{g_{n}(1,1)}{g_{1, n}(1) g_{2, n}(1)}=\frac{1}{(1-a)}\left[\frac{a(1-a)}{a+b}+(1-a)+\frac{(1-a)^{2}}{a+b}\right]=1+\frac{1}{a+b} .
$$

We have to use a bivariate induction to prove the result in e). We state the inductive hypothesis as follows:

Assume that

$$
\lim _{n \rightarrow \infty} \frac{g_{n}\left(s_{1}, s_{2}\right)}{g_{1, n}\left(s_{1}\right) g_{2, n}\left(s_{2}\right)}=1+\frac{1}{a+b}
$$

for all $\left(s_{1}, s_{2}\right)$ such that $s_{1}=1,2, \ldots, X$ and $s_{2}=1,2, \ldots, Y$, together with the results in a), c) and d). Therefore using this hypothesis we need to prove that the result in statement e) holds in the following three cases: 
(i) $(X+1, y)$ such that $y=1,2, \ldots, Y$

(ii) For all $(x, Y+1)$ such that $x=1,2, \ldots, X$

(iii) For $(X+1, Y+1)$.

Note that $X$ and $Y$ should not be confused with random variables. In each case above the argument is similar except that in (i) we use the recursion in (2) whereas in (ii) we use the recursion in formula (3). We will prove the result only in case (i), the other cases follow. Let us fix $y$ such that $y=2,3, \ldots, Y$. Together with the inductive hypothesis and a), c) and d) we also assume that the statement in e) holds for all the pairs $\left(X+1, s_{2}\right)$ such that $s_{2}=1, \ldots, y-1$, then we want to prove the result for $(X+1, y)$. Using the recursion in (2) to evaluate $g_{n}(X+1, y)$ we have

$$
\begin{aligned}
& \lim _{n \rightarrow \infty} \frac{g_{n}(X+1, y)}{g_{1, n}(X+1) g_{2, n}(y)}= \\
& \lim _{n \rightarrow \infty} \frac{1}{1-a p_{1, n}(0) p_{2, n}(0)}\left[\frac{a p_{1, n}(0) \sum_{v=1}^{y} p_{2, n}(v) g_{n}(X+1, y-v)}{g_{1, n}(X+1) g_{2, n}(y)}\right. \\
& \left.\quad+\frac{\sum_{u=1}^{X+1}\left(a+b \frac{u}{X+1}\right) p_{1, n}(u) \sum_{v=0}^{y} p_{2, n}(v) g_{n}(X+1-u, y-v)}{g_{1, n}(X+1) g_{2, n}(y)}\right]
\end{aligned}
$$

To be able to use the results in a), c) and d) we must separate those terms for which one of the entries is zero in the evaluation of $g_{n}$ from the terms where both entries are greater than zero. Doing so we obtain the following result

$$
\begin{aligned}
& \lim _{n \rightarrow \infty} \frac{1}{1-a p_{1, n}(0) p_{2, n}(0)} \times \\
& \quad\left[\frac{a p_{1, n}(0)\left(\sum_{v=1}^{y-1} p_{2, n}(v) g_{n}(X+1, y-v)+p_{2, n}(y) g_{n}(X+1,0)\right)}{g_{1, n}(X+1) g_{2, n}(y)}+\right. \\
& \quad \frac{\sum_{u=1}^{X}\left(a+b \frac{u}{X+1}\right) p_{1, n}(u)\left(\sum_{v=0}^{y-1} p_{2, n}(v) g_{n}(X+1-u, y-v)\right)}{g_{1, n}(X+1) g_{2, n}(y)}+ \\
& \\
& \left.\quad \frac{(a+b) p_{1, n}(X+1)\left(\sum_{v=0}^{y-1} p_{2, n}(v) g_{n}(0, y-v)+p_{2, n}(y) g_{n}(0,0)\right)}{g_{1, n}(X+1) g_{2, n}(y)}\right]
\end{aligned}
$$

Now we can use the same method of multiplying and dividing each term that contains $g_{n}$ by the corresponding product of the marginal distributions. 
Then for those terms where one or both entries is zero the ratio tends to one, and for the ratios where both entries are greater than zero the ratio tends to $1+\frac{1}{(a+b)}$ due to the inductive hypothesis. Therefore, the limit above can be written as follows

$$
\begin{aligned}
& \lim _{n \rightarrow \infty} \frac{g_{n}(X+1, y)}{g_{1, n}(X+1) g_{2, n}(y)}=\lim _{n \rightarrow \infty} \frac{1}{1-a p_{1, n}(0) p_{2, n}(0)} \times \\
& {\left[\left(\frac{a p_{1, n}(0) g_{1, n}(X+1)}{g_{1, n}(X+1)}\right)\left(\frac{\left(1+\frac{1}{a+b}\right) \sum_{v=1}^{y-1} p_{2, n}(v) g_{2, n}(y-v)+p_{2, n}(y) g_{2, n}(0)}{g_{2, n}(y)}\right)+\right.} \\
& +\left(\frac{\sum_{u=1}^{X}\left(a+b \frac{u}{X+1}\right) p_{1, n}(u) g_{1, n}(X+1-u)}{g_{1, n}(X+1)}\right) \times \\
& \left(\frac{\left(1+\frac{1}{a+b}\right) \sum_{v=0}^{y-1} p_{2, n}(v) g_{2, n}(y-v)+p_{2, n}(y) g_{2, n}(0)}{g_{2, n}(y)}\right)+ \\
& \left.+\left(\frac{(a+b) p_{1, n}(X+1) g_{1, n}(0)}{g_{1, n}(X+1)}\right)\left(\frac{p_{2, n}(0) g_{2, n}(y)+\sum_{v=1}^{y} p_{2, n}(v) g_{2, n}(y-v)}{g_{2, n}(y)}\right)\right] .
\end{aligned}
$$

From condition (8) in assumption 6 it follows that $\lim _{n \rightarrow \infty} g_{i, n}(x)=0$ for all $x=1,2,3, \ldots$ and for $i=1,2$. The same result holds for $p_{i, n}(x)$. Using these results we have

$$
\lim _{n \rightarrow \infty} \sum_{v=1}^{y} p_{2, n}(v) g_{2, n}(y-v)=0,
$$

since each term tends to zero. However, when we divide the above sum by $g_{2, n}(y)$ we obtain the following result

$$
\begin{aligned}
& \lim _{n \rightarrow \infty} \frac{\sum_{v=1}^{y} p_{2, n}(v) g_{2, n}(y-v)}{\frac{1}{1-a p_{2, n}(0)} \sum_{v=1}^{y}\left(a+b \frac{v}{y}\right) p_{2, n}(v) g_{2, n}(y-v)}=
\end{aligned}
$$

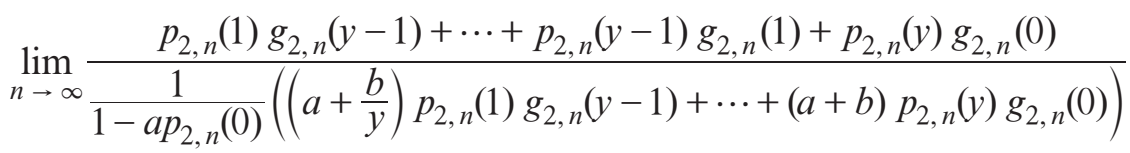

We observe that in the limit above each term tends to zero, however the last term contains $g_{2, n}(0)$ which tends to one as $n$ tends to infinity. Therefore, we divide each term by $p_{2, n}(y)$ and we obtain 
$\lim _{n \rightarrow \infty} \frac{\frac{p_{2, n}(1) g_{2, n}(y-1)}{p_{2, n}(y)}+\cdots+g_{2, n}(0)}{\frac{1}{1-a p_{2, n}(0)}\left(\left(a+\frac{b}{y}\right) \frac{p_{2, n}(1) g_{2, n}(y-1)}{p_{2, n}(y)}+\cdots+(a+b) g_{2, n}(0)\right)}=\frac{1-a}{a+b}$.

The last equality is due to the result in assumption 8 where we assumed that $\lim _{n \rightarrow \infty} \frac{p_{i, n}(x)}{p_{i, n}(y)}=C(x, y)$ which is a constant for $i=1,2$, and $\lim _{n \rightarrow \infty} g_{i, n}(x)=0$ for all $x=1,2, \ldots$. From the discussion above we obtain directly the following results

$$
\begin{aligned}
& \lim _{n \rightarrow \infty} \frac{\sum_{v=1}^{y-1} p_{2, n}(v) g_{2, n}(y-v)}{g_{2, n}(y)}=0 \\
& \lim _{n \rightarrow \infty} \frac{\sum_{u=1}^{X}\left(a+b \frac{u}{X+1}\right) p_{1, n}(u) g_{1, n}(X+1-u)}{g_{1, n}(X+1)}=0
\end{aligned}
$$

$$
\lim _{n \rightarrow \infty} \frac{(a+b) p_{1, n}(X+1) g_{1, n}(0)}{g_{1, n}(X+1)}=1-a
$$

Hence from all the above, we can evaluate the limit in formula (A.1) and we obtain the following result

$$
\lim _{n \rightarrow \infty} \frac{g_{n}(X+1, Y)}{g_{1, n}(X+1) g_{2, n}(Y)}=\frac{1}{1-a}\left[a\left(\frac{1-a}{a+b}\right)+(1-a)\left(1+\frac{1-a}{a+b}\right)\right]=1+\frac{1}{a+b},
$$

which is the result shown in e).

Proposition 2: Suppose that two risks follow assumptions 1 and 3 of Proposition 1. Let $\left\{M_{1, n}\right\}$ and $\left\{M_{2, n}\right\}$ be sequences of real numbers representing the reinsurance retention levels for each portfolio. These sequences are such that the reinsurer's aggregate claim amounts satisfy the condition in (8) and that for each $n$

$$
P\left(S_{1}^{R}\left(M_{1, n}\right)=0\right)=P\left(S_{2}^{R}\left(M_{2, n}\right)=0\right)=\alpha_{n} .
$$

Then, the reinsurance aggregate claim amounts defined in (7) satisfy

$$
\lim _{n \rightarrow \infty} \frac{P\left(S_{1}^{R}\left(M_{1, n}\right)>0, S_{2}^{R}\left(M_{2, n}\right)>0\right)}{P\left(S_{1}^{R}\left(M_{1, n}\right)>0\right) P\left(S_{2}^{R}\left(M_{2, n}\right)>0\right)}=1+\frac{1}{a+b} .
$$

Proof. Note that in this case we do not require that the individual claim amounts are integer-valued random variables. We also do not need that the retention levels are integer numbers. We denote by $p_{i, n}(0)$ the probability that an individual claim amount for the reinsurer is zero and by $g_{i, n}(0)$ the probability that 
the aggregate claim amount for the reinsurer is zero for retention level $M_{i, n}$ for $i=1,2$.

If $n \rightarrow \infty$ then $\alpha_{n} \rightarrow 1^{-}$. We can write the probabilities of being zero in terms of the probability generating function as

$$
g_{i, n}(0)=P\left(S_{i}^{R}\left(M_{i, n}\right)=0\right)=P_{N}\left(p_{i, n}(0)\right)=\alpha_{n} \quad \text { for } i=1,2,
$$

where $P_{N}(t)$ is the probability generating function of the frequency distribution.

Hence, $p_{i, n}(0)=P_{N}^{-1}\left(\alpha_{n}\right)$ for $i=1,2$, provided that the inverse of the probability generating function exists. For the Poisson, the Negative Binomial and the Binomial distributions the inverse of the probability generating function can be written explicitly.

As in part a) of Proposition 1 we can write the joint probability of the aggregate claim amounts being zero as follows

$$
g_{n}(0,0)=P_{N}\left(p_{1, n}(0) p_{2, n}(0)\right)=P_{N}\left(\left(P_{N}^{-1}\left(\alpha_{n}\right)\right)^{2}\right) .
$$

Therefore,

$$
\begin{gathered}
\lim _{n \rightarrow \infty} \frac{P\left(S_{1}^{R}\left(M_{1, n}\right)>0, S_{2}^{R}\left(M_{2, n}\right)>0\right)}{P\left(S_{1}^{R}\left(M_{1, n}\right)>0\right) P\left(S_{2}^{R}\left(M_{2, n}\right)>0\right)}=\lim _{n \rightarrow \infty} \frac{1-g_{1, n}(0)-g_{2, n}(0)+g_{n}(0,0)}{\left(1-g_{1, n}(0)\right)\left(1-g_{2, n}(0)\right)} \\
=\lim _{\alpha_{n} \rightarrow 1^{-}} \frac{1-2 \alpha_{n}+P_{N}\left(\left(P_{N}^{-1}\left(\alpha_{n}\right)\right)^{2}\right)}{\left(1-\alpha_{n}\right)^{2}}=\frac{0}{0} .
\end{gathered}
$$

Applying L'Hospital rule twice the limit above can be calculated as follows

$$
\begin{gathered}
\lim _{\alpha_{n} \rightarrow 1^{-}}\left[2 \frac{d^{2}}{d \alpha_{n}^{2}} P_{N}\left(\left(P_{N}^{-1}\left(\alpha_{n}\right)\right)^{2}\right) \frac{\left(P_{N}^{-1}\left(\alpha_{n}\right)\right)^{2}}{\left(\frac{d}{d \alpha_{n}} P_{N}\left(P_{N}^{-1}\left(\alpha_{n}\right)\right)\right)^{2}}+\right. \\
\left.\frac{1}{\frac{d}{d \alpha_{n}} P_{N}\left(P_{N}^{-1}\left(\alpha_{n}\right)\right)}-\frac{d^{2}}{d \alpha_{n}^{2}} P_{N}\left(P_{N}^{-1}\left(\alpha_{n}\right)\right) \frac{\left(P_{N}^{-1}\left(\alpha_{n}\right)\right)}{\left(\frac{d}{d \alpha_{n}} P_{N}\left(P_{N}^{-1}\left(\alpha_{n}\right)\right)\right)^{2}}\right]
\end{gathered}
$$

From the properties of the probability generating function we have $P_{N}(1)=1$ and therefore $P_{N}^{-1}(1)=1$. Moreover, the probability generating function satisfies $\frac{d}{d t} P_{N}(1)=E[N]$. Therefore, the limit above is given by

$$
\lim _{n \rightarrow \infty} \frac{P\left(S_{1}^{R}\left(M_{1, n}\right)>0, S_{2}^{R}\left(M_{2, n}\right)>0\right)}{P\left(S_{1}^{R}\left(M_{1, n}\right)>0\right) P\left(S_{2}^{R}\left(M_{2, n}\right)>0\right)}=\frac{\frac{d^{2}}{d t^{2}} P_{N}(1)}{(E[N])^{2}}+\frac{1}{E[N]} .
$$


Therefore, by evaluating the limit above with the corresponding values for each of the distributions that belong to Panjer's class it follows that

$$
\lim _{n \rightarrow \infty} \frac{P\left(S_{1}^{R}\left(M_{1, n}\right)>0, S_{2}^{R}\left(M_{2, n}\right)>0\right)}{P\left(S_{1}^{R}\left(M_{1, n}\right)>0\right) P\left(S_{2}^{R}\left(M_{2, n}\right)>0\right)}=1+\frac{1}{a+b} .
$$

ANA J. MATA

CNA Re $e^{1}$

333. S. Wabash Av., 39th floor

Chicago IL 60604

USA

1 The statements, analysis and opinions set forth in this article are solely those of the author and do not reflect the statements, opinions or analysis of any third party, including Continental Casualty Company or any of its subsidiary or affiliated companies. 